\title{
Some New Oscillation Criteria for a Class of Nonlinear Fractional Differential Equations with Damping Term
}

\author{
Bin Zheng and Qinghua Feng \\ School of Science, Shandong University of Technology, Zibo, Shandong 255049, China \\ Correspondence should be addressed to Bin Zheng; zhengbin2601@126.com \\ Received 19 September 2013; Accepted 11 November 2013 \\ Academic Editor: Kai Diethelm
}

Copyright ( 2013 B. Zheng and Q. Feng. This is an open access article distributed under the Creative Commons Attribution License, which permits unrestricted use, distribution, and reproduction in any medium, provided the original work is properly cited.

We are concerned with oscillation of solutions of a class of nonlinear fractional differential equations with damping term. Based on a generalized Riccati function and inequality technique, we establish some new oscillation criteria for it. Some applications are also presented for the established results.

\section{Introduction}

Fractional differential equations are generalizations of classical differential equations of integer order, and one can find their applications in many fields of science and engineering. In the last few decades, research for various aspects of fractional differential equations, for example, the existence, uniqueness, and stability of solutions of fractional differential equations, the numerical methods for fractional differential equations, and so on, has been paid much attention by many authors (e.g., we refer the reader to see [1-8] and the references therein). In these investigations, we notice that very little attention is paid to oscillation of fractional differential equations. Recent results in this direction include Chen's work [9], in which some new oscillation criteria are established for the following fractional differential equation:

$$
\begin{aligned}
& {\left[r(t)\left(D_{-}^{\alpha} x(t)\right)^{\eta}\right]^{\prime}} \\
& \quad-q(t) f\left(\int_{t}^{\infty}(v-t)^{-\alpha} y(v) d v\right)=0, \quad t>0,
\end{aligned}
$$

where $r$ and $q$ are positive functions and $\eta$ is a quotient of two odd positive numbers.
In this paper, we are concerned with oscillation of solutions of the following nonlinear $(2+\alpha)$-order fractional differential equation with damping term:

$$
\begin{aligned}
& \left(a(t)\left[\left(r(t) D_{-}^{\alpha} x(t)\right)^{\prime}\right]^{\gamma}\right)^{\prime}+p(t)\left[\left(r(t) D_{-}^{\alpha} x(t)\right)^{\prime}\right]^{\gamma} \\
& \quad-q(t) f\left(\int_{t}^{\infty}(\xi-t)^{-\alpha} x(\xi) d \xi\right)=0, \quad t \in\left[t_{0}, \infty\right),
\end{aligned}
$$

where $a \in C^{1}\left(\left[t_{0}, \infty\right), \mathbb{R}_{+}\right), r \in C^{2}\left(\left[t_{0}, \infty\right), \mathbb{R}_{+}\right), p, q \in$ $C\left(\left[t_{0}, \infty\right), \mathbb{R}_{+}\right), f \in C(\mathbb{R}, \mathbb{R})$ satisfying $x f(x)>0, f(x) / x^{\gamma} \geq$ $L>0$ for $x \neq 0, \gamma$ is a quotient of two odd positive integers, $\alpha \in(0,1), D_{-}^{\alpha} x(t)$ denotes the Liouville right-sided fractional derivative of order $\alpha$ of $x$, and $D_{-}^{\alpha} x(t)=-(1 / \Gamma(1-$ $\alpha)(d / d t) \int_{t}^{\infty}(\xi-t)^{-\alpha} x(\xi) d \xi$.

A nontrivial solution of (2) is said to be oscillatory if it is neither eventually positive nor eventually negative; otherwise, it is nonoscillatory. Equation (2) is said to be oscillatory in case all its solutions are oscillatory.

Motivated by the idea in [10], we will establish some new oscillation criteria for (2) by a generalized Riccati function and inequality technique in Section 2, and we will present some applications for our results in Section 3. Throughout this paper, $\mathbb{R}$ denotes the set of real numbers and $\mathbb{R}_{+}=$ $(0, \infty)$. For more details about the theory of fractional 
differential equations, we refer the reader to [11-13]. For the sake of convenience, in the rest of this paper, we set $X(t)=$ $\int_{t}^{\infty}(\xi-t)^{-\alpha} x(\xi) d \xi, A(t)=\int_{t_{0}}^{t}(p(s) / a(s)) d s$,

$\theta_{1}(t, a)=\int_{a}^{t} \frac{1}{\left[e^{A(s)} a(s)\right]^{1 / \gamma}} d s, \quad \theta_{2}(t, a)=\int_{a}^{t} \frac{\theta_{1}(s, a)}{r(s)} d s$.

\section{Main Results}

The following lemmas are useful for proving our results.

Lemma 1. Assume that $x$ is a solution of (2). Then, $X^{\prime}(t)=$ $-\Gamma(1-\alpha) D_{-}^{\alpha} x(t)$.

Lemma 2. Assume that $x$ is an eventually positive solution of (2) and

$$
\begin{gathered}
\int_{t_{0}}^{\infty} \frac{1}{\left[e^{A(s)} a(s)\right]^{1 / \gamma}} d s=\infty, \\
\int_{t_{0}}^{\infty} \frac{1}{r(s)} d s=\infty \\
\int_{t_{0}}^{\infty} \frac{1}{r(\xi)} \int_{\xi}^{\infty}\left[\frac{1}{e^{A(\tau)} a(\tau)} \int_{\tau}^{\infty} e^{A(s)} q(s) d s\right]^{1 / \gamma} d \tau d \xi=\infty
\end{gathered}
$$

Then, there exists a sufficiently large $T$ such that

$$
\left(r(t) D_{-}^{\alpha} x(t)\right)^{\prime}<0 \quad \text { on }[T, \infty)
$$

and either $D_{-}^{\alpha} x(t)<0$ on $[T, \infty)$ or $\lim _{t \rightarrow \infty} X(t)=0$.

Proof. Since $x$ is an eventually positive solution of (2), there exists $t_{1}$ such that $x(t)>0$ on $\left[t_{1}, \infty\right)$. So $X(t)>0$ on $\left[t_{1}, \infty\right)$ and we have

$$
\begin{aligned}
\left(e^{A(t)} a(t)\left[\left(r(t) D_{-}^{\alpha} x(t)\right)^{\prime}\right]^{\gamma}\right)^{\prime} & \\
= & e^{A(t)}\left(a(t)\left[\left(r(t) D_{-}^{\alpha} x(t)\right)^{\prime}\right]^{\gamma}\right)^{\prime} \\
& +e^{A(t)} p(t)\left[\left(r(t) D_{-}^{\alpha} x(t)\right)^{\prime}\right]^{\gamma} \\
= & e^{A(t)}\left\{\left(a(t)\left[\left(r(t) D_{-}^{\alpha} x(t)\right)^{\prime}\right]^{\gamma}\right)^{\prime}\right. \\
& \left.\quad+p(t)\left[\left(r(t) D_{-}^{\alpha} x(t)\right)^{\prime}\right]^{\gamma}\right\} \\
= & e^{A(t)} q(t) f(X(t)) \geq L e^{A(t)} q(t) X^{\gamma}(t)>0 .
\end{aligned}
$$

Then, $e^{A(t)} a(t)\left[\left(r(t) D_{-}^{\alpha} x(t)\right)^{\prime}\right]^{\gamma}$ is strictly increasing on $\left[t_{1}, \infty\right)$, and thus $\left(r(t) D_{-}^{\alpha} x(t)\right)^{\prime}$ is eventually of one sign. We claim that $\left(r(t) D_{-}^{\alpha} x(t)\right)^{\prime}<0$ on $\left[t_{2}, \infty\right)$, where $t_{2}>t_{1}$ is sufficiently large. Otherwise, assume there exists a sufficiently large $t_{3}>t_{2}$ such that $\left(r(t) D_{-}^{\alpha} x(t)\right)^{\prime}>0$ on $\left[t_{3}, \infty\right)$. Then, for $t \in\left[t_{3}, \infty\right)$, we have

$$
\begin{aligned}
r(t) & D_{-}^{\alpha} x(t)-r\left(t_{3}\right) D_{-}^{\alpha} x\left(t_{3}\right) \\
= & \int_{t_{3}}^{t} \frac{\left[e^{A(s)} a(s)\right]^{1 / \gamma}\left(r(s) D_{-}^{\alpha} x(s)\right)^{\prime}}{\left[e^{A(s)} a(s)\right]^{1 / \gamma}} d s \\
\geq & {\left[e^{A\left(t_{3}\right)} a\left(t_{3}\right)\right]^{1 / \gamma}\left(r\left(t_{3}\right) D_{-}^{\alpha} x\left(t_{3}\right)\right)^{\prime} } \\
& \times \int_{t_{3}}^{t} \frac{1}{\left[e^{A(s)} a(s)\right]^{1 / \gamma}} d s .
\end{aligned}
$$

By (4), we have

$$
\lim _{t \rightarrow \infty} r(t) D_{-}^{\alpha} x(t)=\infty
$$

which implies, for some sufficiently large $t_{4}>t_{3}, D_{-}^{\alpha} x(t)>0$, $t \in\left[t_{4}, \infty\right)$. By Lemma 1 , we have

$$
\begin{aligned}
X(t)-X\left(t_{4}\right) & =\int_{t_{4}}^{t} X^{\prime}(s) d s \\
& =-\Gamma(1-\alpha) \int_{t_{4}}^{t} D_{-}^{\alpha} x(s) d s \\
& =-\Gamma(1-\alpha) \int_{t_{4}}^{t} \frac{r(s) D_{-}^{\alpha} x(s)}{r(s)} d s \\
& \leq-\Gamma(1-\alpha) r\left(t_{4}\right) D_{-}^{\alpha} x\left(t_{4}\right) \int_{t_{4}}^{t} \frac{1}{r(s)} d s .
\end{aligned}
$$

By (5), we obtain $\lim _{t \rightarrow \infty} X(t)=-\infty$, which contradicts $X(t)>0$ on $\left[t_{1}, \infty\right)$. So $\left(r(t) D_{-}^{\alpha} x(t)\right)^{\prime}<0$ on $\left[t_{2}, \infty\right)$. Thus, $D_{-}^{\alpha} x(t)$ is eventually of one sign. Now, we assume that $D_{-}^{\alpha} x(t)>0, t \in\left[t_{5}, \infty\right)$, for some sufficiently $t_{5}>t_{4}$. Then, by Lemma $1, X^{\prime}(t)<0$ for $t \in\left[t_{5}, \infty\right)$. Since $X(t)>0$, furthermore we have $\lim _{t \rightarrow \infty} X(t)=\beta \geq 0$. We claim that $\beta=0$. Otherwise, assume that $\beta>0$. Then, $X(t) \geq \beta$ on $\left[t_{5}, \infty\right)$, and, for $t \in\left[t_{5}, \infty\right)$, by $(8)$ we have

$$
\begin{aligned}
\left(e^{A(t)} a(t)\left[\left(r(t) D_{-}^{\alpha} x(t)\right)^{\prime}\right]^{\gamma}\right)^{\prime} & \geq L e^{A(t)} q(t) X^{\gamma}(t) \\
& \geq L \beta^{\gamma} e^{A(t)} q(t) .
\end{aligned}
$$

Substituting $t$ with $s$ in (12), an integration for (12) with respect to $s$ from $t$ to $\infty$ yields

$$
\begin{aligned}
& -e^{A(t)} a(t)\left[\left(r(t) D_{-}^{\alpha} x(t)\right)^{\prime}\right]^{\gamma} \\
& \geq-\lim _{t \rightarrow \infty} e^{A(t)} a(t)\left[\left(r(t) D_{-}^{\alpha} x(t)\right)^{\prime}\right]^{\gamma} \\
& +L \beta^{\gamma} \int_{t}^{\infty} e^{A(s)} q(s) d s \\
& >L \beta^{\gamma} \int_{t}^{\infty} e^{A(s)} q(s) d s
\end{aligned}
$$


which means

$$
\left(r(t) D_{-}^{\alpha} x(t)\right)^{\prime}<-L^{1 / \gamma} \beta\left[\frac{1}{e^{A(t)} a(t)} \int_{t}^{\infty} e^{A(s)} q(s) d s\right]^{1 / \gamma} .
$$

Substituting $t$ with $\tau$ in (14), an integration for (14) with respect to $\tau$ from $t$ to $\infty$ yields

$$
\begin{aligned}
- & r(t) D_{-}^{\alpha} x(t) \\
& <-\lim _{t \rightarrow \infty} r(t) D_{-}^{\alpha} x(t) \\
& -L^{1 / \gamma} \beta \int_{t}^{\infty}\left[\frac{1}{e^{A(\tau)} a(\tau)} \int_{\tau}^{\infty} e^{A(s)} q(s) d s\right]^{1 / \gamma} d \tau \\
& <-L^{1 / \gamma} \beta \int_{t}^{\infty}\left[\frac{1}{e^{A(\tau)} a(\tau)} \int_{\tau}^{\infty} e^{A(s)} q(s) d s\right]^{1 / \gamma} d \tau
\end{aligned}
$$

that is,

$$
\begin{aligned}
X^{\prime}(t)< & -L^{1 / \gamma} \Gamma(1-\alpha) \beta \frac{1}{r(t)} \\
& \times \int_{t}^{\infty}\left[\frac{1}{e^{A(\tau)} a(\tau)} \int_{\tau}^{\infty} e^{A(s)} q(s) d s\right]^{1 / \gamma} d \tau .
\end{aligned}
$$

Substituting $t$ with $\xi$ in (16), an integration for (16) with respect to $\xi$ from $t_{5}$ to $t$ yields

$$
\begin{aligned}
X(t) & -X\left(t_{5}\right) \\
< & -L^{1 / \gamma} \Gamma(1-\alpha) \beta \\
\quad & \times \int_{t_{5}}^{t} \frac{1}{r(\xi)} \int_{\xi}^{\infty}\left[\frac{1}{e^{A(\tau)} a(\tau)} \int_{\tau}^{\infty} e^{A(s)} q(s) d s\right]^{1 / \gamma} d \tau d \xi .
\end{aligned}
$$

By (6), one can see that $\lim _{t \rightarrow \infty} X(t)=-\infty$, which is a contradiction. So the proof is complete.

Lemma 3. Assume that $x$ is an eventually positive solution of (2) such that

$$
\left(r(t) D_{-}^{\alpha} x(t)\right)^{\prime}<0, \quad D_{-}^{\alpha} x(t)<0 \quad \text { on }\left[t_{1}, \infty\right)_{\mathbb{T}}
$$

where $t_{1} \geq t_{0}$ is sufficiently large. Then, one has

$$
\begin{gathered}
X^{\prime}(t) \geq-\frac{\Gamma(1-\alpha) \theta_{1}\left(t, t_{1}\right)\left[e^{A(t)} a(t)\right]^{1 / \gamma}\left(r(t) D_{-}^{\alpha} x(t)\right)^{\prime}}{r(t)}, \\
X(t) \geq-\Gamma(1-\alpha) \theta_{2}\left(t, t_{1}\right)\left[e^{A(t)} a(t)\right]^{1 / \gamma}\left(r(t) D_{-}^{\alpha} x(t)\right)^{\prime} .
\end{gathered}
$$

Proof. By Lemma 2, we obtain that $e^{A(t)} a(t)\left[\left(r(t) D_{-}^{\alpha} x(t)\right)^{\prime}\right]^{\gamma}$ is strictly increasing on $\left[t_{1}, \infty\right)$. So

$$
\begin{aligned}
r( & (t) D_{-}^{\alpha} x(t) \leq r(t) D_{-}^{\alpha} x(t)-r\left(t_{1}\right) D_{-}^{\alpha} x\left(t_{1}\right) \\
= & \int_{t_{1}}^{t} \frac{\left[e^{A(s)} a(s)\right]^{1 / \gamma}\left[r(s) D_{-}^{\alpha} x(s)\right]^{\prime}}{\left[e^{A(s)} a(s)\right]^{1 / \gamma}} d s \\
& \leq\left[e^{A(t)} a(t)\right]^{1 / \gamma}\left(r(t) D_{-}^{\alpha} x(t)\right)^{\prime} \int_{t_{1}}^{t} \frac{1}{\left[e^{A(s)} a(s)\right]^{1 / \gamma}} d s \\
& =\theta_{1}\left(t, t_{1}\right)\left[e^{A(t)} a(t)\right]^{1 / \gamma}\left(r(t) D_{-}^{\alpha} x(t)\right)^{\prime} .
\end{aligned}
$$

Using Lemma 1, we obtain that

$$
X^{\prime}(t) \geq-\frac{\Gamma(1-\alpha) \theta_{1}\left(t, t_{1}\right)\left[e^{A(t)} a(t)\right]^{1 / \gamma}\left(r(t) D_{-}^{\alpha} x(t)\right)^{\prime}}{r(t)} .
$$

Then,

$X(t)$

$$
\begin{aligned}
& \geq X(t)-X\left(t_{1}\right) \\
& \geq-\int_{t_{1}}^{t} \frac{\Gamma(1-\alpha) \theta_{1}\left(s, t_{1}\right)\left[e^{A(s)} a(s)\right]^{1 / \gamma}\left(r(t) D_{-}^{\alpha} x(s)\right)^{\prime}}{r(s)} d s \\
& \geq-\Gamma(1-\alpha)\left[e^{A(t)} a(t)\right]^{1 / \gamma}\left(r(t) D_{-}^{\alpha} x(t)\right)^{\prime} \int_{t_{1}}^{t} \frac{\theta_{1}\left(s, t_{1}\right)}{r(s)} d s \\
& =-\Gamma(1-\alpha) \theta_{2}\left(t, t_{1}\right)\left[e^{A(t)} a(t)\right]^{1 / \gamma}\left(r(t) D_{-}^{\alpha} x(t)\right)^{\prime} .
\end{aligned}
$$

Lemma 4 ([14, Theorem 41]). Assume that $A$ and $B$ are nonnegative real numbers. Then,

$$
\lambda A B^{\lambda-1}-A^{\lambda} \leq(\lambda-1) B^{\lambda}, \quad \forall \lambda>1 .
$$

Theorem 5. Assume (4)-(6) hold and there exist two functions $\rho \in C^{1}\left(\left[t_{0}, \infty\right), \mathbb{R}_{+}\right)$and $\eta \in C^{1}\left(\left[t_{0}, \infty\right),[0, \infty)\right)$ such that

$$
\begin{aligned}
\int_{T}^{\infty}\left\{L \rho(s) q(s) e^{A(s)}-\rho(s) \eta^{\prime}(s)\right. \\
+ \\
+\frac{\rho(s) \Gamma(1-\alpha) \theta_{1}(s, T) \eta^{1+(1 / \gamma)}(s)}{r(s)} \\
-\left(\left[(\gamma+1) \eta^{1 / \gamma}(s) \rho(s) \Gamma(1-\alpha) \theta_{1}(s, T)\right.\right. \\
\left.\left.\quad+r(s) \rho^{\prime}(s)\right]^{\gamma+1}\right) \\
\left.\quad \times\left((\gamma+1)^{\gamma+1}\left[\Gamma(1-\alpha) \rho(s) \theta_{1}(s, T)\right]^{\gamma} r(s)\right)^{-1}\right\} d s \\
=\infty,
\end{aligned}
$$

for all sufficiently large $T$. Then, every solution of (2) is oscillatory or satisfies $\lim _{t \rightarrow \infty} X(t)=0$. 
Proof. Assume (2) has a nonoscillatory solution $x$ on $\left[t_{0}, \infty\right)$. Without loss of generality, we may assume $x(t)>0$ on $\left[t_{1}, \infty\right)$, where $t_{1}$ is sufficiently large. By Lemma 2 , we have $\left(r(t) D_{-}^{\alpha} x(t)\right)^{\prime}<0, t \in\left[t_{2}, \infty\right)$, where $t_{2}>t_{1}$ is sufficiently large, and either $D_{-}^{\alpha} x(t)<0$ on $\left[t_{2}, \infty\right)$ or $\lim _{t \rightarrow \infty} X(t)=0$. Define the generalized Riccati function as follows:

$$
\omega(t)=\rho(t)\left\{-\frac{e^{A(t)} a(t)\left[\left(r(t) D_{-}^{\alpha} x(t)\right)^{\prime}\right]^{\gamma}}{X^{\gamma}(t)}+\eta(t)\right\} .
$$

Then, for $t \in\left[t_{2}, \infty\right)$, we have

$\omega^{\prime}(t)$

$$
\begin{aligned}
= & -\rho^{\prime}(t) \frac{e^{A(t)} a(t)\left[\left(r(t) D_{-}^{\alpha} x(t)\right)^{\prime}\right]^{\gamma}}{X^{\gamma}(t)} \\
& +\rho(t)\left\{-\frac{e^{A(t)} a(t)\left[\left(r(t) D_{-}^{\alpha} x(t)\right)^{\prime}\right]^{\gamma}}{X^{\gamma}(t)}\right\} \\
& +\rho^{\prime}(t) \eta(t)+\rho(t) \eta^{\prime}(t) \\
= & -\rho(t)\left\{\left(X^{\gamma}(t)\left(e^{A(t)} a(t)\left[\left(r(t) D_{-}^{\alpha} x(t)\right)^{\prime}\right]^{\gamma}\right)^{\prime}\right.\right. \\
& \left.-\gamma X^{\gamma-1}(t) X^{\prime}(t) e^{A(t)} a(t)\left[\left(r(t) D_{-}^{\alpha} x(t)\right)^{\prime}\right]^{\gamma}\right) \\
= & \left.-\rho\left(X^{2 \gamma}(t)\right)^{-1}\right\}+\frac{\rho^{\prime}(t)}{\rho(t)} \omega(t)+\rho(t) \eta^{\prime}(t) \\
& +\frac{\rho^{\prime}(t)}{\rho(t)} \omega(t)+\rho(t) \eta^{\prime}(t) . \\
+ & \left.\left.+\frac{\rho(t) q(t) e^{A(t)}\left(a(t)\left[\left(r(t) D_{-}^{\alpha} x(t)\right)^{\prime}\right]^{\gamma}\right)^{\prime}}{X^{\gamma}(t)}+X^{A(t)} p(t)\left[\left(r(t) D_{-}^{\alpha} x(t)\right)^{\prime}\right]^{\gamma}\right\}\right) \\
& \times\left(X^{2 \gamma}(t)\right)^{-1} \\
& \left.-\frac{\gamma X^{\gamma-1}(t) X^{\prime}(t) e^{A(t)} a(t)\left[\left(r(t) D_{-}^{\alpha} x(t)\right)^{\prime}\right]^{\gamma}}{X^{2 \gamma}(t)}\right\}
\end{aligned}
$$

By Lemma 3 and the definition of $f$, we get that

$$
\begin{aligned}
\omega^{\prime}(t) \leq & -L \rho(t) q(t) e^{A(t)} \\
- & \left(\gamma \rho(t) \Gamma(1-\alpha) \theta_{1}\left(t, t_{2}\right)\left[e^{A(t)} a(t)\right]^{1 / \gamma}\right. \\
& \left.\times\left(r(t) D_{-}^{\alpha} x(t)\right)^{\prime} a(t) e^{A(t)}\left[\left(r(t) D_{-}^{\alpha} x(t)\right)^{\prime}\right]^{\gamma}\right) \\
\times & \left(r(t) X^{\gamma+1}(t)\right)^{-1} \\
+ & \frac{\rho^{\prime}(t)}{\rho(t)} \omega(t)+\rho(t) \eta^{\prime}(t) \\
= & -L \rho(t) q(t) e^{A(t)}-\frac{\gamma \rho(t) \Gamma(1-\alpha) \theta_{1}\left(t, t_{2}\right)}{r(t)} \\
\times & {\left[-\left(\frac{\omega(t)}{\rho(t)}-\eta(t)\right)\right]^{1+(1 / \gamma)}+\frac{\rho^{\prime}(t)}{\rho(t)} \omega(t)+\rho(t) \eta^{\prime}(t) } \\
=- & L \rho(t) q(t) e^{A(t)}-\frac{\gamma \rho(t) \Gamma(1-\alpha) \theta_{1}\left(t, t_{2}\right)}{r(t)} \\
& \times\left[\frac{\omega(t)}{\rho(t)}-\eta(t)\right]^{1+(1 / \gamma)}+\frac{\rho^{\prime}(t)}{\rho(t)} \omega(t)+\rho(t) \eta^{\prime}(t) .
\end{aligned}
$$

Using the following inequality (see [15, Equation (2.17)])

$$
(u-v)^{1+(1 / \gamma)} \geq u^{1+(1 / \gamma)}+\frac{1}{\gamma} v^{1+(1 / \gamma)}-\left(1+\frac{1}{\gamma}\right) v^{1 / \gamma} u,
$$

we obtain

$$
\begin{aligned}
{\left[\frac{\omega(t)}{\rho(t)}-\eta(t)\right]^{1+(1 / \gamma)} \geq } & \frac{\omega^{1+(1 / \gamma)}(t)}{\rho^{1+(1 / \gamma)}(t)}+\frac{1}{\gamma} \eta^{1+(1 / \gamma)}(t) \\
& -\left(1+\frac{1}{\gamma}\right) \frac{\eta^{1 / \gamma}(t) \omega(t)}{\rho(t)} .
\end{aligned}
$$

A combination of (27) and (29) yields the following:

$\omega^{\prime}(t)$

$$
\begin{aligned}
\leq & -L \rho(t) q(t) e^{A(t)}+\frac{\rho^{\prime}(t)}{\rho(t)} \omega(t)+\rho(t) \eta^{\prime}(t) \\
& -\frac{\gamma \rho(t) \Gamma(1-\alpha) \theta_{1}\left(t, t_{2}\right)}{r(t)} \\
& \times\left[\frac{\omega^{1+(1 / \gamma)}(t)}{\rho^{1+(1 / \gamma)}(t)}+\frac{1}{\gamma} \eta^{1+(1 / \gamma)}(t)-\left(1+\frac{1}{\gamma}\right) \frac{\eta^{1 / \gamma}(t) \omega(t)}{\rho(t)}\right]
\end{aligned}
$$




$$
\begin{aligned}
= & -L \rho(t) q(t) e^{A(t)}+\rho(t) \eta^{\prime}(t) \\
& -\frac{\rho(t) \Gamma(1-\alpha) \theta_{1}\left(t, t_{2}\right) \eta^{1+(1 / \gamma)}(t)}{r(t)} \\
& -\frac{\gamma \rho(t) \Gamma(1-\alpha) \theta_{1}\left(t, t_{2}\right)}{r(t)} \frac{\omega^{1+(1 / \gamma)}(t)}{\rho^{1+(1 / \gamma)}(t)} \\
& +\frac{(\gamma+1) \eta^{1 / \gamma}(t) \rho(t) \Gamma(1-\alpha) \theta_{1}\left(t, t_{2}\right)+r(t) \rho^{\prime}(t)}{r(t) \rho(t)} \omega(t) .
\end{aligned}
$$

Setting

$$
\begin{gathered}
\lambda=1+\frac{1}{\gamma}, \\
A^{\lambda}=\frac{\gamma \rho(t) \Gamma(1-\alpha) \theta_{1}\left(t, t_{2}\right)}{r(t)} \frac{\omega^{1+(1 / \gamma)}(t)}{\rho^{1+(1 / \gamma)}(t)}, \\
B^{\lambda-1} \\
=\gamma^{1 /(\gamma+1)} \\
\times \frac{(\gamma+1) \eta^{1 / \gamma}(t) \rho(t) \Gamma(1-\alpha) \theta_{1}\left(t, t_{2}\right)+r(t) \rho^{\prime}(t)}{(\gamma+1)\left[\Gamma(1-\alpha) \rho(t) \theta_{1}\left(t, t_{2}\right)\right]^{\gamma /(\gamma+1)} r^{1 /(\gamma+1)}(t)} .
\end{gathered}
$$

Using Lemma 4 in (30), we get that

$\omega^{\prime}(t)$

$$
\begin{aligned}
\leq & -L \rho(t) q(t) e^{A(t)}+\rho(t) \eta^{\prime}(t) \\
& -\frac{\rho(t) \Gamma(1-\alpha) \theta_{1}\left(t, t_{2}\right) \eta^{1+(1 / \gamma)}(t)}{r(t)} \\
& +\frac{\left[(\gamma+1) \eta^{1 / \gamma}(t) \rho(t) \Gamma(1-\alpha) \theta_{1}\left(t, t_{2}\right)+r(t) \rho^{\prime}(t)\right]^{\gamma+1}}{(\gamma+1)^{\gamma+1}\left[\Gamma(1-\alpha) \rho(t) \theta_{1}\left(t, t_{2}\right)\right]^{\gamma} r(t)} .
\end{aligned}
$$

Substituting $t$ with $s$ in (32), an integration for (32) with respect to $s$ from $t_{2}$ to $t$ yields

$$
\begin{aligned}
\int_{t_{2}}^{t}\{ & L \rho(s) q(s) e^{A(s)}-\rho(s) \eta^{\prime}(s) \\
+ & \frac{\rho(s) \Gamma(1-\alpha) \theta_{1}\left(s, t_{2}\right) \eta^{1+(1 / \gamma)}(s)}{r(s)} \\
- & \left(\left[(\gamma+1) \eta^{1 / \gamma}(s) \rho(s) \Gamma(1-\alpha) \theta_{1}\left(s, t_{2}\right)\right.\right. \\
& \left.\left.+r(s) \rho^{\prime}(s)\right]^{\gamma+1}\right) \\
& \left.\times\left((\gamma+1)^{\gamma+1}\left[\Gamma(1-\alpha) \rho(s) \theta_{1}\left(s, t_{2}\right)\right]^{\gamma} r(s)\right)^{-1}\right\} d s \\
\leq & \omega\left(t_{2}\right)-\omega(t) \leq \omega\left(t_{2}\right),
\end{aligned}
$$

which contradicts (24). So the proof is complete.
Theorem 6. Assume (4)-(6) hold and, for all sufficiently large $T$,

$$
\begin{aligned}
\int_{T}^{\infty}\left\{L \rho(s) q(s) e^{A(s)}-\rho(s) \eta^{\prime}(s)\right. \\
+\frac{\gamma \rho(s)[\Gamma(1-\alpha)]^{\gamma} \theta_{1}(s, T) \theta_{2}^{\gamma-1}(s, T) \eta^{2}(s)}{r(s)} \\
-\left(\left\{2 \gamma \rho(s) \eta(s)[\Gamma(1-\alpha)]^{\gamma} \theta_{1}(s, T) \theta_{2}^{\gamma-1}(s, T)\right.\right. \\
\left.\left.\quad+r(s) \rho^{\prime}(s)\right\}^{2}\right) \\
\left.\quad \times\left(4 \gamma[\Gamma(1-\alpha)]^{\gamma} \theta_{1}(s, T) \theta_{2}^{\gamma-1}(s, T) r(s) \rho(s)\right)^{-1}\right\} d s \\
=\infty,
\end{aligned}
$$

where $\rho$ and $\eta$ are defined as in Theorem 5. Then, every solution of (2) is oscillatory or satisfies $\lim _{t \rightarrow \infty} X(t)=0$.

Proof. Assume (2) has a nonoscillatory solution $x$ on $\left[t_{0}, \infty\right)$. Without loss of generality, we may assume $x(t)>0$ on $\left[t_{1}, \infty\right)$, where $t_{1}$ is sufficiently large. By Lemma 2 , we have $\left(r(t) D_{-}^{\alpha} x(t)\right)^{\prime}<0, t \in\left[t_{2}, \infty\right)$, where $t_{2}>t_{1}$ is sufficiently large, and either $D_{-}^{\alpha} x(t)<0$ on $\left[t_{2}, \infty\right)$ or $\lim _{t \rightarrow \infty} X(t)=0$. Let $\omega(t)$ be defined as in Theorem 5 . Proceeding as in Theorem 5, we obtain (26). By Lemma 3, we have the following observation:

$$
\frac{X^{\prime}(t)}{X(t)}
$$

$$
\geq-\frac{\Gamma(1-\alpha) \theta_{1}\left(t, t_{2}\right)\left[e^{A(t)} a(t)\right]^{1 / \gamma}\left(r(t) D_{-}^{\alpha} x(t)\right)^{\prime}}{r(t) X(t)}
$$$$
=-\frac{\Gamma(1-\alpha) \theta_{1}\left(t, t_{2}\right)\left[e^{A(t)} a(t)\right]^{1 / \gamma}\left(r(t) D_{-}^{\alpha} x(t)\right)^{\prime}}{r(t) X^{\gamma}(t)}
$$$$
\times X^{\gamma-1}(t)
$$$$
\geq-\frac{\Gamma(1-\alpha) \theta_{1}\left(t, t_{2}\right)\left[e^{A(t)} a(t)\right]^{1 / \gamma}\left(r(t) D_{-}^{\alpha} x(t)\right)^{\prime}}{r(t) X^{\gamma}(t)}
$$$$
\times\left\{-\Gamma(1-\alpha) \theta_{2}\left(t, t_{2}\right)\left[e^{A(t)} a(t)\right]^{1 / \gamma}\left(r(t) D_{-}^{\alpha} x(t)\right)^{\prime}\right\}^{\gamma-1}
$$$$
=-\frac{[\Gamma(1-\alpha)]^{\gamma} \theta_{1}\left(t, t_{2}\right) \theta_{2}^{\gamma-1}\left(t, t_{2}\right)}{r(t)}
$$$$
\times\left\{\frac{e^{A(t)} a(t)\left[\left(r(t) D_{-}^{\alpha} x(t)\right)^{\prime}\right]^{\gamma}}{X^{\gamma}(t)}\right\} .
$$ 
Using (35) in (26) we get that

$\omega^{\prime}(t)$

$$
\begin{aligned}
\leq & -L \rho(t) q(t) e^{A(t)}-\frac{\gamma \rho(t)[\Gamma(1-\alpha)]^{\gamma} \theta_{1}\left(t, t_{2}\right) \theta_{2}^{\gamma-1}\left(t, t_{2}\right)}{r(t)} \\
& \times\left\{\frac{e^{A(t)} a(t)\left[\left(r(t) D_{-}^{\alpha} x(t)\right)^{\prime}\right]^{\gamma}}{X^{\gamma}(t)}\right\} \\
+ & \frac{\rho^{\prime}(t)}{\rho(t)} \omega(t)+\rho(t) \eta^{\prime}(t) \\
= & -L \rho(t) q(t) e^{A(t)}-\frac{\gamma \rho(t)[\Gamma(1-\alpha)]^{\gamma} \theta_{1}\left(t, t_{2}\right) \theta_{2}^{\gamma-1}\left(t, t_{2}\right)}{r(t)} \\
& \times\left[\frac{\omega(t)}{\rho(t)}-\eta(t)\right]^{2}+\frac{\rho^{\prime}(t)}{\rho(t)} \omega(t)+\rho(t) \eta^{\prime}(t) \\
& +\left(\left\{2 \gamma \rho(t) \eta(t)[\Gamma(1-\alpha)]^{\gamma} \theta_{1}\left(t, t_{2}\right) \theta_{2}^{\gamma-1}\left(t, t_{2}\right)\right.\right. \\
& -L \rho(t) q(t) e^{A(t)} \\
& +\frac{\gamma \rho(t)[\Gamma(1-\alpha)]^{\gamma} \theta_{1}\left(t, t_{2}\right) \theta_{2}^{\gamma-1}\left(t, t_{2}\right) \eta^{2}(t)}{r(t)} \\
& -\frac{\gamma \rho(t)[\Gamma(1-\alpha)]^{\gamma} \theta_{1}\left(t, t_{2}\right) \theta_{2}^{\gamma-1}\left(t, t_{2}\right) \eta^{2}(t)}{r(t)} \\
& +\frac{\gamma \gamma \rho(t) \eta(t)[\Gamma(1-\alpha)]^{\gamma} \theta_{1}\left(t, t_{2}\right) \theta_{2}^{\gamma-1}\left(t, t_{2}\right)+r(t) \rho^{\prime}(t)}{r(t) \rho(t)} \\
& \frac{\gamma(1-\alpha)]^{\gamma} \theta_{1}\left(t, t_{2}\right) \theta_{2}^{\gamma-1}\left(t, t_{2}\right)}{r(t) \rho(t)} \omega^{2}(t)
\end{aligned}
$$

Substituting $t$ with $s$ in (36), an integration for (36) with respect to $s$ from $t_{2}$ to $t$ yields

$$
\begin{aligned}
\int_{t_{2}}^{t}\{ & L \rho(s) q(s) e^{A(s)}-\rho(s) \eta^{\prime}(s) \\
+ & \frac{\gamma \rho(s)[\Gamma(1-\alpha)]^{\gamma} \theta_{1}\left(s, t_{2}\right) \theta_{2}^{\gamma-1}\left(s, t_{2}\right) \eta^{2}(s)}{r(s)} \\
- & \left(\left\{2 \gamma \rho(s) \eta(s)[\Gamma(1-\alpha)]^{\gamma} \theta_{1}\left(s, t_{2}\right) \theta_{2}^{\gamma-1}\left(s, t_{2}\right)\right.\right. \\
& \left.\left.+r(s) \rho^{\prime}(s)\right\}^{2}\right)
\end{aligned}
$$

$$
\begin{aligned}
& \left.\times\left(4 \gamma[\Gamma(1-\alpha)]^{\gamma} \theta_{1}\left(s, t_{2}\right) \theta_{2}^{\gamma-1}\left(s, t_{2}\right) r(s) \rho(s)\right)^{-1}\right\} d s \\
\leq & \omega\left(t_{2}\right)-\omega(t) \leq \omega\left(t_{2}\right),
\end{aligned}
$$

which contradicts (34). So the proof is complete.

Theorem 7. Define $\mathbb{D}=\left\{(t, s) \mid t \geq s \geq t_{0}\right\}$. Assume (4)-(6) hold and there exists a function $H \in C^{1}(\mathbb{D}, \mathbb{R})$ such that

$$
H(t, t)=0, \quad \text { for } t \geq t_{0}, \quad H(t, s)>0, \quad \text { for } t>s \geq t_{0} \text {, }
$$

$H$ has a nonpositive continuous partial derivative $H_{s}^{\prime}(t, s)$, and

$$
\begin{aligned}
& \lim _{t \rightarrow \infty} \sup \frac{1}{H\left(t, t_{0}\right)} \\
& \times\left\{\int_{t_{0}}^{t} H(t, s)\right. \\
& \times\left\{L \rho(s) q(s) e^{A(s)}-\rho(s) \eta^{\prime}(s)\right. \\
& +\frac{\rho(s) \Gamma(1-\alpha) \theta_{1}(s, T) \eta^{1+(1 / \gamma)}(s)}{r(s)} \\
& -\left(\left[(\gamma+1) \eta^{1 / \gamma}(s) \rho(s) \Gamma(1-\alpha) \theta_{1}(s, T)\right.\right. \\
& \left.\left.\quad+r(s) \rho^{\prime}(s)\right]^{\gamma+1}\right) \\
& \times\left((\gamma+1)^{\gamma+1}\left[\Gamma(1-\alpha) \rho(s) \theta_{1}(s, T)\right]^{\gamma}\right. \\
& \left.\left.\times r(s))^{-1}\right\} d s\right\} \\
& =\infty, \quad
\end{aligned}
$$

for all sufficiently large $T$, where $\rho$ and $\eta$ are defined as in Theorem 5. Then, every solution of (2) is oscillatory or satisfies $\lim _{t \rightarrow \infty} X(t)=0$.

Proof. Assume (2) has a nonoscillatory solution $x$ on $\left[t_{0}, \infty\right)$. Without loss of generality, we may assume $x(t)>0$ on $\left[t_{1}, \infty\right)$, where $t_{1}$ is sufficiently large. By Lemma 2 , we have either $D_{-}^{\alpha} x(t)<0$ on $\left[t_{2}, \infty\right)$, for some sufficiently large $t_{2}>t_{1}$, or $\lim _{t \rightarrow \infty} X(t)=0$. Now we assume $D_{-}^{\alpha} x(t)<0$. Let $\omega(t)$ be defined as in Theorem 5. By (32), we have

$$
\begin{aligned}
& L \rho(t) q(t) e^{A(t)}-\rho(t) \eta^{\prime}(t) \\
& +\frac{\rho(t) \Gamma(1-\alpha) \theta_{1}\left(t, t_{2}\right) \eta^{1+(1 / \gamma)}(t)}{r(t)} \\
& \quad-\frac{\left[(\gamma+1) \eta^{1 / \gamma}(t) \rho(t) \Gamma(1-\alpha) \theta_{1}\left(t, t_{2}\right)+r(t) \rho^{\prime}(t)\right]^{\gamma+1}}{(\gamma+1)^{\gamma+1}\left[\Gamma(1-\alpha) \rho(t) \theta_{1}\left(t, t_{2}\right)\right]^{\gamma} r(t)} \\
& \leq-\omega^{\prime}(t) .
\end{aligned}
$$


Journal of Applied Mathematics

7

Substituting $t$ with $s$ in (40), multiplying both sides by $H(t, s)$, and then integrating with respect to $s$ from $t_{2}$ to $t$ yield

$$
\begin{aligned}
& \int_{t_{2}}^{t} H(t, s) \\
& \times\left\{L \rho(s) q(s) e^{A(s)}-\rho(s) \eta^{\prime}(s)\right. \\
& \quad+\frac{\rho(s) \Gamma(1-\alpha) \theta_{1}\left(s, t_{2}\right) \eta^{1+(1 / \gamma)}(s)}{r(s)} \\
& \quad-\left(\left[(\gamma+1) \eta^{1 / \gamma}(s) \rho(s) \Gamma(1-\alpha) \theta_{1}\left(s, t_{2}\right)\right.\right. \\
& \left.\left.\quad+r(s) \rho^{\prime}(s)\right]^{\gamma+1}\right) \\
& \left.\quad \times\left((\gamma+1)^{\gamma+1}\left[\Gamma(1-\alpha) \rho(s) \theta_{1}\left(s, t_{2}\right)\right]^{\gamma} r(s)\right)^{-1}\right\} d s \\
& \leq-\int_{t_{2}}^{t} H(t, s) \omega^{\prime}(s) d s=H\left(t, t_{2}\right) \omega\left(t_{2}\right) \\
& +\int_{t_{2}}^{t} H_{s}^{\prime}(t, s) \omega(s) \Delta s \\
& \leq H\left(t, t_{2}\right) \omega\left(t_{2}\right) \leq H\left(t, t_{0}\right) \omega\left(t_{2}\right) .
\end{aligned}
$$

Then

$$
\begin{aligned}
& \int_{t_{0}}^{t} H(t, s) \\
& \times\left\{L \rho(s) q(s) e^{A(s)}-\rho(s) \eta^{\prime}(s)\right. \\
& +\frac{\rho(s) \Gamma(1-\alpha) \theta_{1}\left(s, t_{2}\right) \eta^{1+(1 / \gamma)}(s)}{r(s)} \\
& -\left(\left[(\gamma+1) \eta^{1 / \gamma}(s) \rho(s) \Gamma(1-\alpha) \theta_{1}\left(s, t_{2}\right)\right.\right. \\
& \left.\left.+r(s) \rho^{\prime}(s)\right]^{\gamma+1}\right) \\
& \left.\times\left((\gamma+1)^{\gamma+1}\left[\Gamma(1-\alpha) \rho(s) \theta_{1}\left(s, t_{2}\right)\right]^{\gamma} r(s)\right)^{-1}\right\} d s \\
& =\int_{t_{0}}^{t_{2}} H(t, s) \\
& \times\left\{L \rho(s) q(s) e^{A(s)}-\rho(s) \eta^{\prime}(s)\right. \\
& +\frac{\rho(s) \Gamma(1-\alpha) \theta_{1}\left(s, t_{2}\right) \eta^{1+(1 / \gamma)}(s)}{r(s)} \\
& -\left(\left[(\gamma+1) \eta^{1 / \gamma}(s) \rho(s) \Gamma(1-\alpha) \theta_{1}\left(s, t_{2}\right)\right.\right. \\
& \left.\left.+r(s) \rho^{\prime}(s)\right]^{\gamma+1}\right) \\
& \left.\times\left((\gamma+1)^{\gamma+1}\left[\Gamma(1-\alpha) \rho(s) \theta_{1}\left(s, t_{2}\right)\right]^{\gamma} r(s)\right)^{-1}\right\} d s
\end{aligned}
$$

$$
\begin{aligned}
& +\int_{t_{2}}^{t} H(t, s) \\
& \times\left\{L \rho(s) q(s) e^{A(s)}-\rho(s) \eta^{\prime}(s)\right. \\
& +\frac{\rho(s) \Gamma(1-\alpha) \theta_{1}\left(s, t_{2}\right) \eta^{1+(1 / \gamma)}(s)}{r(s)} \\
& -\left(\left[(\gamma+1) \eta^{1 / \gamma}(s) \rho(s) \Gamma(1-\alpha) \theta_{1}\left(s, t_{2}\right)\right.\right. \\
& \left.\left.+r(s) \rho^{\prime}(s)\right]^{\gamma+1}\right) \\
& \left.\times\left((\gamma+1)^{\gamma+1}\left[\Gamma(1-\alpha) \rho(s) \theta_{1}\left(s, t_{2}\right)\right]^{\gamma} r(s)\right)^{-1}\right\} d s \\
& \leq H\left(t, t_{0}\right) \omega\left(t_{2}\right)+H\left(t, t_{0}\right) \\
& \times \int_{t_{0}}^{t_{2}} \mid L \rho(s) q(s) e^{A(s)}-\rho(s) \eta^{\prime}(s) \\
& +\frac{\rho(s) \Gamma(1-\alpha) \theta_{1}\left(s, t_{2}\right) \eta^{1+(1 / \gamma)}(s)}{r(s)} \\
& -\left(\left[(\gamma+1) \eta^{1 / \gamma}(s) \rho(s) \Gamma(1-\alpha) \theta_{1}\left(s, t_{2}\right)\right.\right. \\
& \left.\left.+r(s) \rho^{\prime}(s)\right]^{\gamma+1}\right) \\
& \times\left((\gamma+1)^{\gamma+1}\left[\Gamma(1-\alpha) \rho(s) \theta_{1}\left(s, t_{2}\right)\right]^{\gamma} r(s)\right)^{-1} \mid d s .
\end{aligned}
$$

So

$$
\begin{aligned}
& \lim _{t \rightarrow \infty} \sup \frac{1}{H\left(t, t_{0}\right)} \\
& \times\left\{\int_{t_{0}}^{t} H(t, s)\right. \\
& \times\left\{L \rho(s) q(s) e^{A(s)}-\rho(s) \eta^{\prime}(s)\right. \\
& +\frac{\rho(s) \Gamma(1-\alpha) \theta_{1}\left(s, t_{2}\right) \eta^{1+(1 / \gamma)}(s)}{r(s)} \\
& \quad-\left(\left[(\gamma+1) \eta^{1 / \gamma}(s) \rho(s) \Gamma(1-\alpha) \theta_{1}\left(s, t_{2}\right)\right.\right. \\
& \left.\left.\quad+r(s) \rho^{\prime}(s)\right]^{\gamma+1}\right) \\
& \quad\left((\gamma+1)^{\gamma+1}\left[\Gamma(1-\alpha) \rho(s) \theta_{1}\left(s, t_{2}\right)\right]^{\gamma}\right. \\
& \left.\left.\quad \times r(s))^{-1}\right\} d s\right\} \\
& +\int_{t_{0}}^{t_{2}} L \rho(s) q(s) e^{A(s)}-\rho(s) \eta^{\prime}(s) \\
& \leq \omega\left(t_{2}\right) \quad
\end{aligned}
$$




$$
\begin{aligned}
& +\frac{\rho(s) \Gamma(1-\alpha) \theta_{1}\left(s, t_{2}\right) \eta^{1+(1 / \gamma)}(s)}{r(s)} \\
& -\left(\left[(\gamma+1) \eta^{1 / \gamma}(s) \rho(s) \Gamma(1-\alpha) \theta_{1}\left(s, t_{2}\right)\right.\right. \\
& \left.\left.\quad+r(s) \rho^{\prime}(s)\right]^{\gamma+1}\right) \\
& \times\left((\gamma+1)^{\gamma+1}\left[\Gamma(1-\alpha) \rho(s) \theta_{1}\left(s, t_{2}\right)\right]^{\gamma} r(s)\right)^{-1} \mid d s
\end{aligned}
$$$$
<\infty
$$

which contradicts (39). So the proof is complete.

Theorem 8. Let $H, \rho$, and $\eta$ be defined as in Theorem 7. If (4)(6) hold and

$$
\begin{aligned}
\lim _{t \rightarrow \infty} \sup & \frac{1}{H\left(t, t_{0}\right)} \\
\times \int_{t_{0}}^{t} H(t, s) & \\
\times\left\{L \rho(s) q(s) e^{A(s)}-\rho(s) \eta^{\prime}(s)\right. & \\
& +\frac{\gamma \rho(s)[\Gamma(1-\alpha)]^{\gamma} \theta_{1}(s, T) \theta_{2}^{\gamma-1}(s, T) \eta^{2}(s)}{r(s)} \\
& -\left(\left\{2 \gamma \rho(s) \eta(s)[\Gamma(1-\alpha)]^{\gamma} \theta_{1}(s, T) \theta_{2}^{\gamma-1}(s, T)\right.\right. \\
& \left.\left.+r(s) \rho^{\prime}(s)\right\}^{2}\right) \\
= & \left.\times\left(4 \gamma[\Gamma(1-\alpha)]^{\gamma} \theta_{1}(s, T) \theta_{2}^{\gamma-1}(s, T) r(s) \rho(s)\right)^{-1}\right\} d s
\end{aligned}
$$

for all sufficiently large $T$, then every solution of (2) is oscillatory or satisfies $\lim _{t \rightarrow \infty} X(t)=0$.

Proof. Assume (2) has a nonoscillatory solution $x$ on $\left[t_{0}, \infty\right)$. Without loss of generality, we may assume $x(t)>0$ on $\left[t_{1}, \infty\right)$, where $t_{1}$ is sufficiently large. By Lemma 2 , we have either $D_{-}^{\alpha} x(t)<0$ on $\left[t_{2}, \infty\right)$, for some sufficiently large $t_{2}>t_{1}$, or $\lim _{t \rightarrow \infty} X(t)=0$. Now, we assume $D_{-}^{\alpha} x(t)<0$. Let $\omega(t)$ be defined as in Theorem 5. By (36), we have

$$
\begin{aligned}
& L \rho(t) q(t) e^{A(t)}-\rho(t) \eta^{\prime}(t) \\
& +\frac{\gamma \rho(t)[\Gamma(1-\alpha)]^{\gamma} \theta_{1}\left(t, t_{2}\right) \theta_{2}^{\gamma-1}\left(t, t_{2}\right) \eta^{2}(t)}{r(t)}
\end{aligned}
$$

$$
\begin{aligned}
& -\frac{\left\{2 \gamma \rho(t) \eta(t)[\Gamma(1-\alpha)]^{\gamma} \theta_{1}\left(t, t_{2}\right) \theta_{2}^{\gamma-1}\left(t, t_{2}\right)+r(t) \rho^{\prime}(t)\right\}^{2}}{4 \gamma[\Gamma(1-\alpha)]^{\gamma} \theta_{1}\left(t, t_{2}\right) \theta_{2}^{\gamma-1}\left(t, t_{2}\right) r(t) \rho(t)} \\
& \leq-\omega^{\prime}(t) .
\end{aligned}
$$

Substituting $t$ with $s$ in (45), multiplying both sides by $H(t, s)$, and then integrating with respect to $s$ from $t_{2}$ to $t$ yield

$$
\begin{aligned}
& \int_{t_{2}}^{t} H(t, s) \\
& \times\left\{L \rho(s) q(s) e^{A(s)}-\rho(s) \eta^{\prime}(s)\right. \\
& \quad+\frac{\gamma \rho(s)[\Gamma(1-\alpha)]^{\gamma} \theta_{1}\left(s, t_{2}\right) \theta_{2}^{\gamma-1}\left(s, t_{2}\right) \eta^{2}(s)}{r(s)} \\
& \quad-\left(\left\{2 \gamma \rho(s) \eta(s)[\Gamma(1-\alpha)]^{\gamma} \theta_{1}\left(s, t_{2}\right) \theta_{2}^{\gamma-1}\left(s, t_{2}\right)\right.\right. \\
& \left.\left.\quad+r(s) \rho^{\prime}(t)\right\}^{2}\right) \\
& \left.\quad \times\left(4 \gamma[\Gamma(1-\alpha)]^{\gamma} \theta_{1}\left(s, t_{2}\right) \theta_{2}^{\gamma-1}\left(s, t_{2}\right) r(s) \rho(s)\right)^{-1}\right\} d s \\
& \leq-\int_{t_{2}}^{t} H(t, s) \omega^{\prime}(s) d s=H\left(t, t_{2}\right) \omega\left(t_{2}\right) \\
& +\int_{t_{2}}^{t} H_{s}^{\prime}(t, s) \omega(s) \Delta s \\
& \leq H\left(t, t_{2}\right) \omega\left(t_{2}\right) \leq H\left(t, t_{0}\right) \omega\left(t_{2}\right) .
\end{aligned}
$$

Then, similar to the process of Theorem 7 , we get that

$$
\begin{aligned}
& \lim _{t \rightarrow \infty} \sup \frac{1}{H\left(t, t_{0}\right)} \\
& \times\left\{\int_{t_{0}}^{t} H(t, s)\right. \\
& \times\left\{L \rho(s) q(s) e^{A(s)}-\rho(s) \eta^{\prime}(s)\right. \\
&+\frac{\gamma \rho(s)[\Gamma(1-\alpha)]^{\gamma} \theta_{1}\left(s, t_{2}\right) \theta_{2}^{\gamma-1}\left(s, t_{2}\right) \eta^{2}(s)}{r(s)} \\
& \quad-\left(\left\{2 \gamma \rho(s) \eta(s)[\Gamma(1-\alpha)]^{\gamma} \theta_{1}\left(s, t_{2}\right)\right.\right. \\
&\left.\left.\quad \times \theta_{2}^{\gamma-1}\left(s, t_{2}\right)+r(s) \rho^{\prime}(s)\right\}^{2}\right) \\
& \times\left(4 \gamma[\Gamma(1-\alpha)]^{\gamma} \theta_{1}\left(s, t_{2}\right) \theta_{2}^{\gamma-1}\right. \\
&\left.\left.\left.\quad \times\left(s, t_{2}\right) r(s) \rho(s)\right)^{-1}\right\} d s\right\}<\infty
\end{aligned}
$$

which contradicts (44). So the proof is complete. 
Remark 9. In Theorems 7 and 8 , if we take $H(t, s)$ for some special functions such as $(t-s)^{m}$ or $\ln (t / s)$, then we can obtain some corollaries, which are omitted here.

Remark 10. The established oscillation criteria for (2) above are new results so far in the literature to the best of our knowledge.

\section{Applications}

In this section, we will present some applications for the established results above.

Example 1. Consider the following:

$$
\begin{aligned}
& \left(t^{5 / 3}\left[\left(D_{-}^{\alpha} x(t)\right)^{\prime}\right]^{5 / 3}\right)^{\prime}+t^{-8 / 3}\left[\left(D_{-}^{\alpha} x(t)\right)^{\prime}\right]^{5 / 3} \\
& -t^{-8 / 3}\left[M+e^{\int_{t}^{\infty}(\xi-t)^{-\alpha} x(\xi) d \xi}\right] \\
& \quad \times\left(\int_{t}^{\infty}(\xi-t)^{-\alpha} x(\xi) d \xi\right)^{5 / 3}=0, \quad t \in[2, \infty),
\end{aligned}
$$

where $M>0$ is a constant.

We have in (2) $\gamma=5 / 3, a(t)=t^{5 / 3}, p(t)=q(t)=t^{-8 / 3}$, $f(x)=x^{5 / 3}\left[e^{x}+M\right], r(t)=1, t_{0}=2$. Then, $f(x) / x^{\gamma} \geq$ $M=L$. Moreover, $1 \leq e^{A(t)}=e^{\int_{2}^{t}(p(s) / a(s)) d s}=e^{\int_{2}^{t} s^{-13 / 3} d s}=$ $e^{-(3 / 10)\left[t^{-10 / 3}-2^{-10 / 3}\right]} \leq e$. Then, we have

$$
\begin{gathered}
\int_{t_{0}}^{\infty} \frac{1}{\left[e^{A(s)} a(s)\right]^{1 / \gamma}} d s=\int_{2}^{\infty} \frac{1}{\left[e^{A(s)} a(s)\right]^{3 / 5}} d s \\
\geq e^{-3 / 5} \int_{2}^{\infty} \frac{1}{s} d s=\infty, \\
\int_{t_{0}}^{\infty} \frac{1}{r(s)} d s=\infty .
\end{gathered}
$$

Furthermore,

$$
\begin{aligned}
& \int_{t_{0}}^{\infty} \frac{1}{r(\xi)} \int_{\xi}^{\infty}\left[\frac{1}{e^{A(\tau)} a(\tau)} \int_{\tau}^{\infty} e^{A(s)} q(s) d s\right]^{1 / \gamma} d \tau d \xi \\
& \quad=\int_{2}^{\infty} \int_{\xi}^{\infty}\left[\frac{1}{e^{A(\tau)} \tau^{5 / 3}} \int_{\tau}^{\infty} e^{A(s)} s^{-8 / 3} d s\right]^{3 / 5} d \tau d \xi \\
& \geq e^{-3 / 5} \int_{2}^{\infty} \int_{\xi}^{\infty}\left[\frac{1}{\tau^{5 / 3}} \int_{\tau}^{\infty} s^{-8 / 3} d s\right]^{3 / 5} d \tau d \xi \\
&=\left(\frac{5 e}{3}\right)^{-3 / 5} \int_{2}^{\infty}\left[\int_{\xi}^{\infty} \frac{1}{\tau^{2}} d \tau\right] d \xi=\left(\frac{5 e}{3}\right)^{-3 / 5} \int_{2}^{\infty} \frac{1}{\xi} d \xi=\infty
\end{aligned}
$$

On the other hand, for a sufficiently large $T$, we have

$$
\begin{aligned}
\theta_{1}(t, T) & =\int_{T}^{t} \frac{1}{\left[e^{A(s)} a(s)\right]^{1 / \gamma}} d s \\
& =\int_{T}^{t} \frac{1}{\left[e^{A(s)} s^{5 / 3}\right]^{3 / 5}} d s \geq e^{-3 / 5} \int_{T}^{t} \frac{1}{s} d s \longrightarrow \infty .
\end{aligned}
$$

So we can take $T^{*}>T$ such that $\theta_{1}(t, T)>1$ for $t \in\left[T^{*}, \infty\right)$. Taking $\rho(t)=t^{5 / 3}$ and $\eta(t)=0$ in (24), we get that

$$
\begin{aligned}
\int_{T}^{\infty}\{L \rho(s) q(s) & \left.-\frac{\left[r(s) \rho^{\prime}(s)\right]^{\gamma+1}}{(\gamma+1)^{\gamma+1}\left[\Gamma(1-\alpha) \rho(s) \theta_{1}(s, T)\right]^{\gamma} r(s)}\right\} d s \\
= & \int_{T}^{\infty}\left\{M-\left(\frac{5}{8}\right)^{8 / 3} \frac{1}{\left[\Gamma(1-\alpha) \theta_{1}(s, T)\right]^{5 / 3}}\right\} \frac{1}{s} d s \\
= & \int_{T}^{T^{*}}\left\{M-\left(\frac{5}{8}\right)^{8 / 3} \frac{1}{\left[\Gamma(1-\alpha) \theta_{1}(s, T)\right]^{5 / 3}}\right\} \frac{1}{s} d s \\
& +\int_{T^{*}}^{\infty}\left\{M-\left(\frac{5}{8}\right)^{8 / 3} \frac{1}{\left[\Gamma(1-\alpha) \theta_{1}(s, T)\right]^{5 / 3}}\right\} \frac{1}{s} d s \\
\geq & \int_{T}^{T^{*}}\left\{M-\left(\frac{5}{8}\right)^{8 / 3} \frac{1}{\left[\Gamma(1-\alpha) \theta_{1}(s, T)\right]^{5 / 3}}\right\} \frac{1}{s} d s \\
& +\int_{T^{*}}^{t}\left\{M-\left(\frac{5}{8}\right)^{8 / 3} \frac{1}{\left.[\Gamma(1-\alpha)]^{5 / 3}\right\} \frac{1}{s} d s} \longrightarrow \infty, \longrightarrow\right.
\end{aligned}
$$

provided that $M>(5 / 8)^{8 / 3}\left(1 /[\Gamma(1-\alpha)]^{5 / 3}\right)$. So (4)-(6) and (24) all hold, and by Theorem 5 we deduce that every solution of (48) is oscillatory or satisfies $\lim _{t \rightarrow \infty} X(t)=0$ under the condition $M>(5 / 8)^{8 / 3}\left(1 /[\Gamma(1-\alpha)]^{5 / 3}\right)$.

Example 2. Consider the following:

$$
\begin{aligned}
& \left(t^{3}\left[\left(D_{-}^{\alpha} x(t)\right)^{\prime}\right]^{3}\right)^{\prime}+t^{-4}\left[\left(D_{-}^{\alpha} x(t)\right)^{\prime}\right]^{3} \\
& -M t^{-4}\left[\int_{t}^{\infty}(\xi-t)^{-\alpha} x(\xi) d \xi\right]^{3}=0, \quad t \in[2, \infty),
\end{aligned}
$$

where $\alpha \in(0,1)$ and $M>0$ is a constant.

We have in (2) $\gamma=3, a(t)=t^{3}, p(t)=q(t)=t^{-4}, f(x)=$ $M x^{3}, r(t)=1, t_{0}=2$. Then, $f(x) / x^{\gamma} \geq M=L$. Moreover, $1 \leq e^{A(t)}=e^{\int_{2}^{t}(p(s) / a(s)) d s}=e^{\int_{2}^{t} s^{-7} d s}=e^{-(1 / 6)\left[t^{-6}-2^{-6}\right]} \leq e^{1 / 384}$. Then, we have

$$
\begin{gathered}
\int_{t_{0}}^{\infty} \frac{1}{\left[e^{A(s)} a(s)\right]^{1 / \gamma}} d s=\int_{2}^{\infty} \frac{1}{\left[e^{A(s)} a(s)\right]^{1 / 3}} d s \\
\geq e^{-1 / 1152} \int_{2}^{\infty} \frac{1}{s} d s=\infty, \\
\int_{t_{0}}^{\infty} \frac{1}{r(s)} d s=\infty .
\end{gathered}
$$


Furthermore,

$$
\begin{aligned}
& \int_{t_{0}}^{\infty} \frac{1}{r(\xi)} \int_{\xi}^{\infty}\left[\frac{1}{e^{A(\tau)} a(\tau)} \int_{\tau}^{\infty} e^{A(s)} q(s) d s\right]^{1 / \gamma} d \tau d \xi \\
& \quad=\int_{2}^{\infty} \int_{\xi}^{\infty}\left[\frac{1}{e^{A(\tau)} \tau^{3}} \int_{\tau}^{\infty} e^{A(s)} s^{-4} d s\right]^{1 / 3} d \tau d \xi \\
& \geq e^{-1 / 1152} \int_{2}^{\infty} \int_{\xi}^{\infty}\left[\frac{1}{\tau^{3}} \int_{\tau}^{\infty} s^{-4} d s\right]^{1 / 3} d \tau d \xi \\
& =\frac{e^{-1 / 1152}}{\sqrt[3]{3}} \int_{2}^{\infty}\left[\int_{\xi}^{\infty} \frac{1}{\tau^{2}} d \tau\right] d \xi=\frac{e^{-1 / 1152}}{\sqrt[3]{3}} \int_{2}^{\infty} \frac{1}{\xi} d \xi=\infty
\end{aligned}
$$

On the other hand, for a sufficiently large $T$, we have

$$
\begin{aligned}
\theta_{1}(t, T) & =\int_{T}^{t} \frac{1}{\left[e^{A(s)} a(s)\right]^{1 / \gamma}} d s \\
& =\int_{T}^{t} \frac{1}{\left[e^{A(s)} s^{3}\right]^{1 / 3}} d s \geq e^{-1 / 1152} \int_{T}^{t} \frac{1}{s} d s \longrightarrow \infty
\end{aligned}
$$

So we can take $T^{*}>T$ such that $\theta_{1}(t, T)>1$ for $t \in\left[T^{*}, \infty\right)$. Taking $\rho(t)=t^{3}, \eta(t)=0, H(t, s)=t-s$ in (39), we get that

$$
\begin{aligned}
& \lim _{t \rightarrow \infty} \sup \frac{1}{t-t_{0}} \\
& \times\left\{\int_{t_{0}}^{t}(t-s)\right. \\
& \times\{L \rho(s) q(s) \\
& =\lim _{t \rightarrow \infty} \sup \frac{1}{t-2} \\
& \quad \times\left\{\int_{2}^{t}(t-s)\right. \\
& \left.\quad \times\left\{M-\left(\frac{3}{4}\right)^{4} \frac{\left[r(s) \rho^{\prime}(s)\right]^{\gamma+1}}{\left[\Gamma(1-\alpha) \theta_{1}(s, T)\right]^{3}}\right\} \frac{1}{s} d s\right\}
\end{aligned}
$$

$$
\begin{aligned}
& =\lim _{t \rightarrow \infty} \sup \frac{1}{t-2} \\
& \quad \times\left\{\int_{2}^{T^{*}}(t-s)\right. \\
& \quad \times\left\{M-\left(\frac{3}{4}\right)^{4} \frac{1}{\left[\Gamma(1-\alpha) \theta_{1}(s, T)\right]^{3}}\right\} \frac{1}{s} d s \\
& +\int_{T^{*}}^{t}(t-s) \\
& \left.\geq \lim _{t \rightarrow \infty} \sup ^{\frac{1}{t-2}}\left\{M-\left(\frac{3}{4}\right)^{4} \frac{1}{\left[\Gamma(1-\alpha) \theta_{1}(s, T)\right]^{3}}\right\} \frac{1}{s} d s\right\} \\
& \quad \times\left\{\int_{2}^{T^{*}}(t-s)\left\{M-\left(\frac{3}{4}\right)^{4} \frac{1}{[\Gamma(1-\alpha)]^{3}}\right\} \frac{1}{s} d s\right. \\
& \left.+\int_{T^{*}}^{t}(t-s)\left\{M-\left(\frac{3}{4}\right)^{4} \frac{1}{[\Gamma(1-\alpha)]^{3}}\right\} \frac{1}{s} d s\right\}=\infty,
\end{aligned}
$$

provided that $M>(3 / 4)^{4}\left(1 /[\Gamma(1-\alpha)]^{3}\right)$. So $(4)-(6)$ and (39) all hold, and by Theorem 7 we deduce that every solution of (48) is oscillatory or satisfies $\lim _{t \rightarrow \infty} X(t)=0$ under the condition $M>(3 / 4)^{4}\left(1 /[\Gamma(1-\alpha)]^{3}\right)$.

\section{Conflict of Interests}

The authors declare that there is no conflict of interests regarding the publication of this paper.

\section{Acknowledgments}

This work was partially supported by the Natural Science Foundation of Shandong Province (in China) (Grant no. ZR2013AQ009) and the Doctoral Initializing Foundation of Shandong University of Technology (in China) (Grant no. 4041-413030).

\section{References}

[1] A. Saadatmandi and M. Dehghan, "A new operational matrix for solving fractional-order differential equations," Computers \& Mathematics with Applications, vol. 59, no. 3, pp. 1326-1336, 2010.

[2] Y. Zhou, F. Jiao, and J. Li, "Existence and uniqueness for $p$-type fractional neutral differential equations," Nonlinear Analysis: Theory, Methods \& Applications, vol. 71, no. 7-8, pp. 2724-2733, 2009.

[3] L. Galeone and R. Garrappa, "Explicit methods for fractional differential equations and their stability properties," Journal of Computational and Applied Mathematics, vol. 228, no. 2, pp. 548-560, 2009. 
[4] J. C. Trigeassou, N. Maamri, J. Sabatier, and A. Oustaloup, "A Lyapunov approach to the stability of fractional differential equations," Signal Processing, vol. 91, pp. 437-445, 2011.

[5] W. Deng, "Smoothness and stability of the solutions for nonlinear fractional differential equations," Nonlinear Analysis: Theory, Methods \& Applications, vol. 72, no. 3-4, pp. 1768-1777, 2010.

[6] F. Ghoreishi and S. Yazdani, "An extension of the spectral Tau method for numerical solution of multi-order fractional differential equations with convergence analysis," Computers \& Mathematics with Applications, vol. 61, no. 1, pp. 30-43, 2011.

[7] J. T. Edwards, N. J. Ford, and A. C. Simpson, "The numerical solution of linear multi-term fractional differential equations; systems of equations," Journal of Computational and Applied Mathematics, vol. 148, no. 2, pp. 401-418, 2002.

[8] M. Muslim, "Existence and approximation of solutions to fractional differential equations," Mathematical and Computer Modelling, vol. 49, no. 5-6, pp. 1164-1172, 2009.

[9] D.-X. Chen, "Oscillation criteria of fractional differential equations," Advances in Difference Equations, vol. 2012, article 33, pp. $1-18,2012$.

[10] Q. Zhang and F. Qiu, "Oscillation theorems for second-order half-linear delay dynamic equations with damping on time scales," Computers \& Mathematics with Applications, vol. 62, no. 11, pp. 4185-4193, 2011.

[11] I. Podlubny, Fractional Differential Equations, Academic Press, San Diego, Calif, USA, 1999.

[12] A. A. Kilbas, H. M. Srivastava, and J. J. Trujillo, Theory and Applications of Fractional Differential Equations, vol. 204, Elsevier, Amsterdam, The Netherlands, 2006.

[13] S. Das, Functional Fractional Calculus for System Identification and Controls, Springer, New York, NY, USA, 2008.

[14] G. H. Hardy, J. E. Littlewood, and G. Pólya, Inequalities, Cambridge University Press, Cambridge, UK, 1988.

[15] T. S. Hassan, "Oscillation of third order nonlinear delay dynamic equations on time scales," Mathematical and Computer Modelling, vol. 49, no. 7-8, pp. 1573-1586, 2009. 


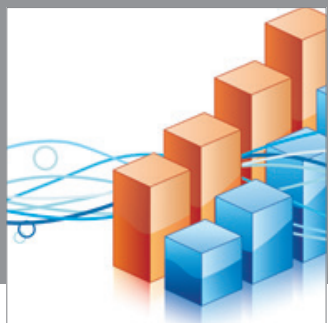

Advances in

Operations Research

mansans

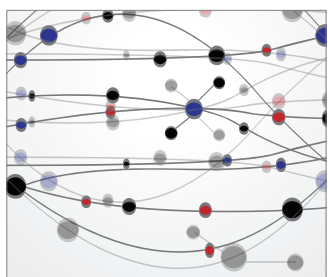

The Scientific World Journal
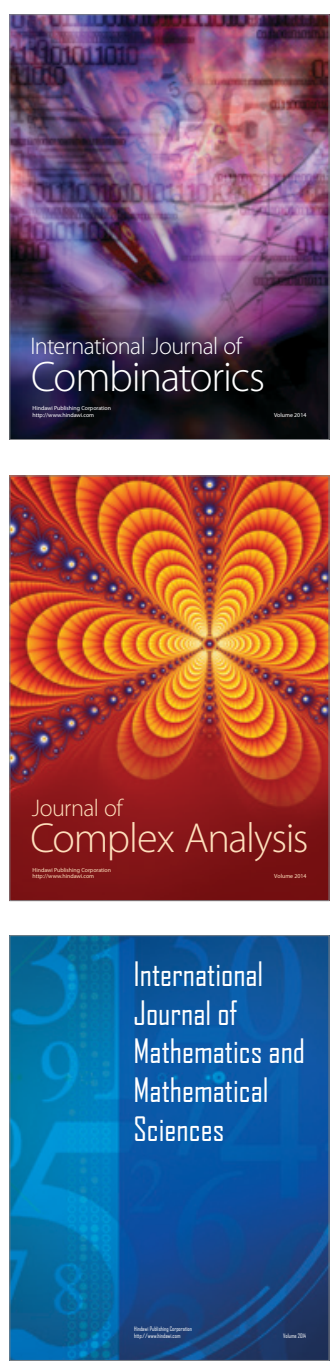
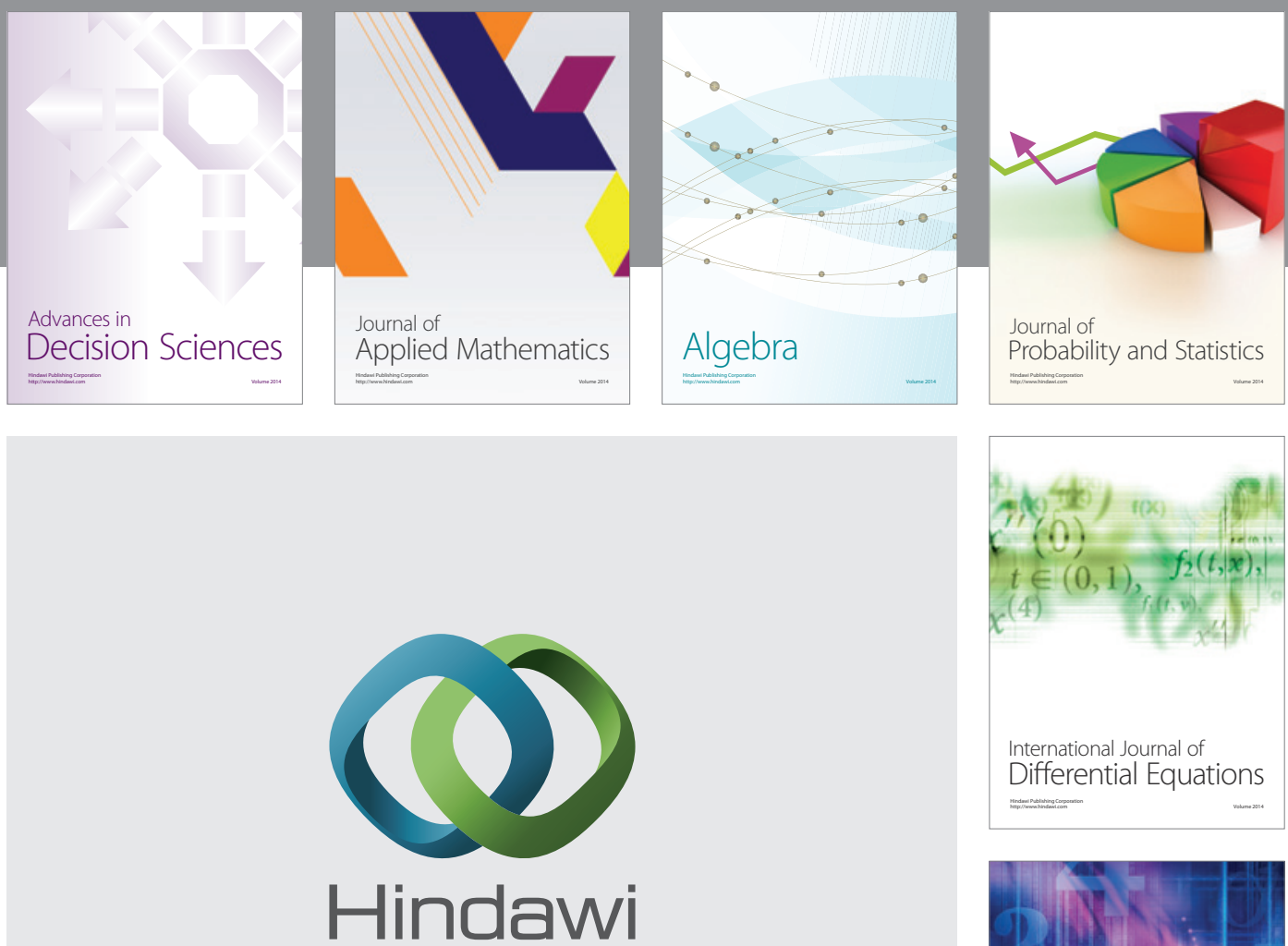

Submit your manuscripts at http://www.hindawi.com
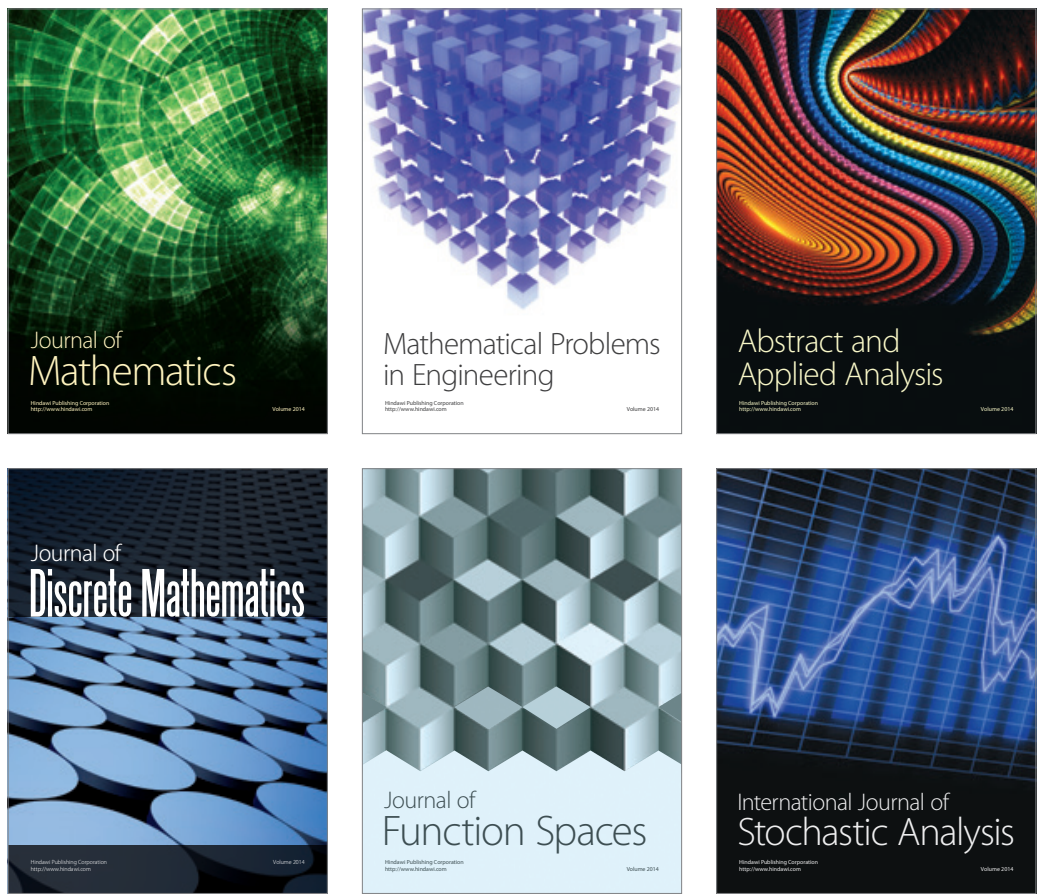

Journal of

Function Spaces

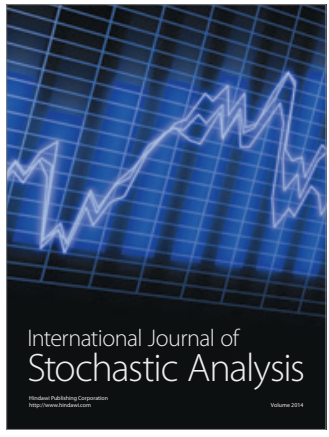

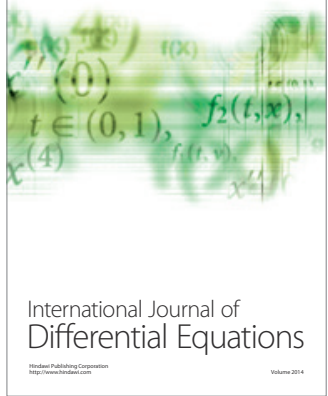
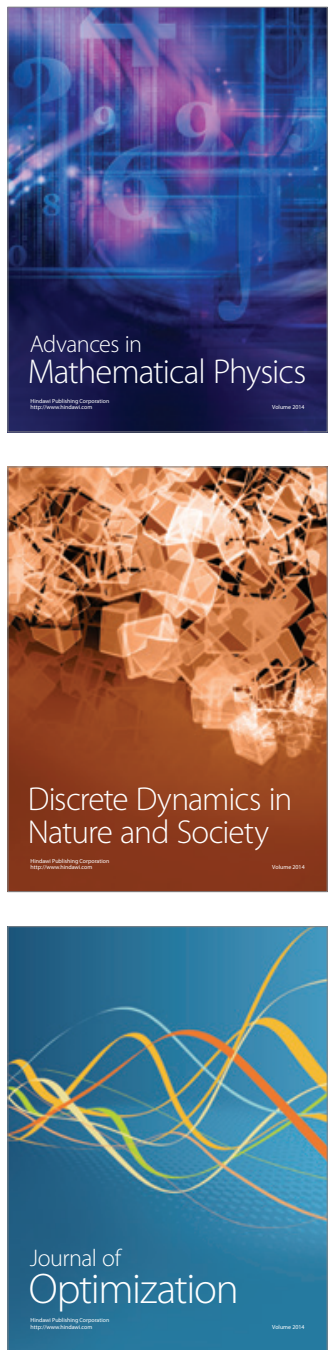